\title{
Microwave phase locking of Josephson-junction fluxon oscillators
}

\author{
Salerno, M.; Samuelsen, Mogens Rugholm; Filatrella, G.; Pagano, S.; Parmentier, R. D.
}

Published in:

Physical Review B

Link to article, DOI:

10.1103/PhysRevB.41.6641

Publication date:

1990

Document Version

Publisher's PDF, also known as Version of record

Link back to DTU Orbit

Citation (APA):

Salerno, M., Samuelsen, M. R., Filatrella, G., Pagano, S., \& Parmentier, R. D. (1990). Microwave phase locking of Josephson-junction fluxon oscillators. Physical Review B, 41(10), 6641-6654.

https://doi.org/10.1103/PhysRevB.41.6641

\section{General rights}

Copyright and moral rights for the publications made accessible in the public portal are retained by the authors and/or other copyright owners and it is a condition of accessing publications that users recognise and abide by the legal requirements associated with these rights.

- Users may download and print one copy of any publication from the public portal for the purpose of private study or research.

- You may not further distribute the material or use it for any profit-making activity or commercial gain

- You may freely distribute the URL identifying the publication in the public portal 


\title{
Microwave phase locking of Josephson-junction fluxon oscillators
}

\author{
M. Salerno \\ Department of Theoretical Physics, University of Salerno, I-84100 Salerno, Italy \\ M. R. Samuelsen \\ Physics Laboratory I, The Technical University of Denmark, DK-2800 Lyngby, Denmark \\ G. Filatrella \\ Department of Physics, University of Salerno, I-84100 Salerno, Italy \\ S. Pagano \\ Institute of Cybernetics of the Consiglio Nazionale delle Ricerche, I-80072 Arco Felice, Napoli, Italy \\ R. D. Parmentier \\ Department of Physics, University of Salerno, I-84100 Salerno, Italy \\ (Received 1 June 1989; revised manuscript received 10 October 1989)

\begin{abstract}
Application of the classic McLaughlin-Scott soliton perturbation theory to a Josephson-junction fluxon subjected to a microwave field that interacts with the fluxon only at the junction boundaries reduces the problem of phase locking of the fluxon oscillation to the study of a two-dimensional functional map. Phase-locked states correspond to fixed points of the map. For junctions of in-line geometry, the existence and stability of such fixed points can be studied analytically. Study of overlap-geometry junctions requires the numerical inversion of a functional equation, but the results are qualitatively very similar. The map predicts significantly different behaviors for locking at odd and even subharmonic frequencies and at superharmonic frequencies. It also gives indications regarding hysteresis in the current-voltage characteristic, the existence of zero-crossing steps, and a description of the locking process in the frequency domain. The principal merit of the map is that it captures much of the experimental phenomenology at a very low computational cost.
\end{abstract}

\section{INTRODUCTION}

Interest in the phenomenon of phase locking of fluxon oscillations in long Josephson tunnel junctions has recently been stimulated by the possibility of employing arrays of such long junctions as local oscillators in integrated superconductive microwave or millimeter-wave receivers for radioastronomy and space communications. ${ }^{1}$ Phase locking in arrays of point Josephson junctions has been under active study for some time; ${ }^{2}$ such arrays described mathematically by systems of nonlinear, coupled ordinary differential equations (ODE's). Fluxon oscillations in long junctions, on the other hand, are described by partial differential equations (PDE's); ${ }^{3}$ this fact has perhaps tended to retard the theoretical study of phase locking in arrays of such junctions, even though the earlier experimental studies also go back a number of years. ${ }^{4}$

For the experimentalist, interest in fluxon oscillations in long junctions derives largely from the fact that the intrinsic linewidth of the radiation emitted from a single fluxon oscillator is quite narrow; relative linewidths as small as $10^{-7}$ have been measured in the $X$ band. $^{5}$ A significant obstacle to incorporating fluxon oscillators into practical receivers has been that the power available from a single oscillator has so far proved to be too small to be practically useful. It is this fact that has suggested the construction and study of arrays of long-junction os- cillators.

A complete mathematical model of an array of fluxon oscillators consists of a system of nonlinear coupled PDE's. Results have been reported on systems of two coupled fluxon oscillators, coupled through a resistance, ${ }^{6}$ an inductance, ${ }^{7}$ and a capacitance, ${ }^{8}$ but the detailed study of larger arrays still presents notable difficulties, also because the exact nature of the coupling between the individual oscillators in such arrays remains to be clarified. A simpler, but related, problem is to study the interaction of a single oscillator with a fixed, external microwave field. Scheuermann et al. ${ }^{9}$ and Cirillo and Lloyd ${ }^{10}$ have described experimental measurements of the behavior of a single long-junction oscillator in an external field. Their results show that a study of this simpler system can shed significant light on the more complicated problem of the interaction between multiple junctions in an array.

Our theoretical approach to the study of this simpler problem is based on the perturbation analysis of fluxon dynamics pioneered by McLaughlin and Scott. ${ }^{11}$ This perturbation analysis was extended to the interaction with microwave fields by Chang. ${ }^{12}$ Our work takes this approach one step further, reducing the problem to the study of a discrete, two-dimensional functional map, in which, for example, a stable phase-locked fluxon oscillation corresponds to one (or more) stable fixed point(s) in the map. Obviously, this approach enormously reduces 
the computational effort required to study the problem. In spite of this drastic simplification, however, the approach remarkably succeeds in capturing, at least qualitatively, much of the experimentally observed phenomenology. A brief, preliminary description of the phaselocking map has been presented elsewhere. ${ }^{13}$

\section{MATHEMATICAL MODEL}

The electrodynamics of a Josephson junction is described by a $(2+1)$-dimensional sine-Gordon PDE for the phase difference $\phi$ between the junction electrodes. In normalized form this equation can be written ${ }^{14}$

$$
\phi_{x x}+\phi_{y y}-\phi_{t t}-\sin \phi=\alpha \phi_{t}-\beta\left(\phi_{x x t}+\phi_{y y t}\right)
$$

(we ignore here the so-called $\cos \phi$ term). Here, $x$ and $y$ are spatial coordinates normalized to the Josephson penetration length $\lambda_{J}, t$ is time normalized to the inverse of the Josephson angular plasma frequency $\omega_{0}$, the term in $\alpha$ represents shunt dissipation caused by quasiparticle tunneling (here, as usual, assumed ohmic), the term in $\beta$ represents dissipation caused by the surface resistance of the superconducting junction electrodes, and subscripts denote partial derivatives. Details of the normalizations may be found in Ref. 14. Boundary conditions for Eq. (1), at $x=0, L$ and $y=0, W$, are determined, respectively, by the $y$ and $x$ components of the magnetic field to which the junction is subjected. ${ }^{15}$

In practice, a long, narrow junction structure is normally employed, i.e., $L>1$ and $W \ll<1$, which permits the reduction of Eq. (1) to a $1+1$ dimensional model. The exact form of the reduced model depends on the physical geometry of the junction. The two junction geometries that have so far received the most experimental attention are the so-called overlap and in-line geometries. ${ }^{15}$ For the overlap geometry, assuming a constant (physical) bias current $I_{\text {bias }}$ in the $y$ direction and a spatially uniform but time-varying magnetic field applied in the $y$ direction, the model reduces to ${ }^{14}$

$$
\phi_{x x}-\phi_{t t}-\sin \phi=\alpha \phi_{t}-\beta \phi_{x x t}-\gamma,
$$

with the boundary conditions

$$
\phi_{x}(0, t)+\beta \phi_{x t}(0, t)=\phi_{x}(L, t)+\beta \phi_{x t}(L, t)=\eta .
$$

Here, $\gamma \equiv I_{\text {bias }} / J_{0} L \lambda_{J}$, where $J_{0}$ is the (physical) maximum pair-current density per unit length in the $x$ direction, and $\boldsymbol{\eta}$ is a normalized measure of the $y$ component of the external magnetic field. A condition for the validity of the reduction is: ${ }^{14} \gamma W^{2} / 8 \ll<$.

The essential difference for the in-line geometry is that the bias current enters through the boundary conditions, since $I_{\text {bias }}$ is now applied in the $x$ direction, rather than as a term in the PDE. Thus the PDE becomes

$$
\phi_{x x}-\phi_{t t}-\sin \phi=\alpha \phi_{t}-\beta \phi_{x x t},
$$

and the boundary conditions become

$$
\begin{aligned}
& \phi_{x}(0, t)+\beta \phi_{x t}(0, t)=-\kappa+\eta, \\
& \phi_{x}(L, t)+\beta \phi_{x t}(L, t)=+\kappa+\eta,
\end{aligned}
$$

where $\kappa \equiv I_{\text {bias }} / 2 J_{0} / \lambda_{J}$.

Our theoretical approach to the study of phase locking in long junctions is based on two fundamental hypotheses. (i) The influence of an external microwave field on a long-junction oscillator is felt only through the boundary conditions, not through the PDE, i.e., we assume the experimental conditions to be such that the microwave field does not significantly penetrate the interior of the junction. (ii) The dynamics of fluxons in the interior of a junction is adequately described by the perturbation analysis of McLaughlin and Scott. ${ }^{11}$

The essential motivation for hypothesis (i) is the observation $^{16}$ that in the numerical integration of Eqs. (2), an ac driver introduced through the term $\eta$ is much more "effective" in generating phase-locked states than is a similar driver introduced through a spatially uniform, but time-dependent term $\gamma$. Moreover, as pointed out by Chang, ${ }^{12}$ there are physical reasons for supposing that a microwave field cannot penetrate deeply into the junction. The motivation for hypothesis (ii) is the welldemonstrated success of the perturbation approach in describing various aspects of fluxon dynamics.

Furthermore, purely for computational convenience, we make the following simplifying assumptions: (a) The parameter $\beta$ in Eqs. (2a) and (3a) is set to zero. Consideration of a $\beta \neq 0$ would render the analysis somewhat, but not overwhelmingly, more complicated. (b) The junction length $L$ is large compared with unity so that we can employ as a solution ansatz a form appropriate to the infinite-length limit. A modification of this assumption, involving a perturbation treatment of phase shift and power dissipation effects at the junction boundaries ${ }^{17}$ is discussed in Sec. VII later. (c) Only dynamic states involving a single fluxon (or antifluxon) are considered.

\section{PERTURBATION ANALYSIS}

We follow herein the procedure first reported by McLaughlin and Scott ${ }^{11}$ and further elaborated by Christiansen and Olsen, ${ }^{18}$ by Levring et al. ${ }^{19}$ and by Chang. ${ }^{12}$

\section{A. Overlap geometry}

In the infinite-length limit, a fluxon solution of Eq. (2a) is well characterized by its momentum $P_{f}$, defined as

$$
P_{f}=-\int_{-\infty}^{+\infty} \phi_{x} \phi_{t} d x
$$

From Eqs. (2a) and (4), the equation of motion for a single fluxon (with $\beta=0$ ) is

$$
\frac{d P_{f}}{d t}=-\alpha P_{f}+2 \pi \gamma
$$

For the pure sine-Gordon equation $(\alpha=\gamma=0)$ in the infinite-length limit, the single fluxon solution has the well-known form 


$$
\phi_{0}(x, t)=4 \arctan \left\{\exp \left[-\left(x-u t-x_{0}\right) /\left(1-u^{2}\right)^{1 / 2}\right]\right\}
$$

in which $x_{0}$ is the initial position of the fluxon, and $u$ is its (constant) velocity of propagation. In this case, from Eqs. (4) and (6), the momentum may be calculated explicitly as

$$
P_{f 0}=8 u /\left(1-u^{2}\right)^{1 / 2}
$$

The first essential ingredient of the perturbation analysis is to assume that if $\alpha$ and $\gamma$ in Eq. (2a) are sufficiently small, the perturbed fluxon may be well described by the form

$$
\phi_{p}(x, t)=4 \arctan \left(\exp \left\{-[x-X(t)] /\left[1-u^{2}(t)\right]^{1 / 2}\right\}\right),
$$

in which the fluxon position $X(t)$ is given by

$$
X(t)=x_{0}+\int_{0}^{t} u(\tau) d \tau
$$

The dynamics of the fluxon in the interior of the junction may then be calculated by inserting this form, through Eq. (7), into Eq. (5). One simple, well-known result of this procedure is that, for given $\alpha$ and $\gamma$, there exists an equilibrium velocity, called $u_{\infty}$, for which energy input and dissipation are exactly balanced. This velocity is found from the stationary solution of Eq. (5), with $P_{f}$ given by Eq. (7). The result is

$$
u_{\infty}=\left[1+(4 \alpha / \pi \gamma)^{2}\right]^{-1 / 2} \text {. }
$$

Equation (5) may be conveniently integrated in terms of the quantity $z(t)$, defined as

$$
z(t) \equiv u(t) /\left[1-u^{2}(t)\right]^{1 / 2}=P_{f 0}(t) / 8
$$

The approximation involved here is the identification of $P_{f}(t)$ in Eq. (5) with $P_{f 0}(t)$ in Eq. (11). The result of the integration is

$$
z(t)=z_{\infty}+\left(z_{0}-z_{\infty}\right) \exp (-\alpha t),
$$

in which $z_{0}$ is the initial value of $z$, and $z_{\infty}$ corresponds to $u_{\infty}$ in Eq. (10). The fluxon trajectory may then be found by combining Eqs. (9), (11), and (12). The result is ${ }^{19}$

$$
\begin{aligned}
X(t)= & x_{0}+u_{\infty} t-\frac{1}{\alpha} \ln \left[\frac{z+\left(z^{2}+1\right)^{1 / 2}}{z_{0}+\left(z_{0}^{2}+1\right)^{1 / 2}}\right] \\
& -\frac{u_{\infty}}{\alpha} \ln \left(\frac{1+z_{\infty} \cdot z_{0}+\left(z_{\infty}^{2}+1\right)^{1 / 2}\left(z_{0}^{2}+1\right)^{1 / 2}}{1+z \cdot z_{\infty}+\left(z^{2}+1\right)^{1 / 2}\left(z_{\infty}^{2}+1\right)^{1 / 2}}\right) .
\end{aligned}
$$

(We note parenthetically here that Eq. (12) of Ref. 19 contains a printing error.)

The second essential ingredient of the analysis is the treatment of the boundary condition, Eq. (2b). Following Levring et al. ${ }^{19}$ we observe that during a reflection from a boundary, due to Eq. ( $2 b$ ) a fluxon undergoes an energy variation $\Delta H_{f}$, given by

$$
\Delta H_{f}= \pm 4 \pi \eta
$$

For constant $\eta$, this variation is positive at (say) the lefthand boundary and negative at (say) the right-hand boundary. To relate this energy variation to the fluxon trajectory $X(t)$, we recall that the energy of a solution of the pure sine-Gordon equation in the infinite-length limit is

$$
H=\int_{-\infty}^{+\infty}\left[\frac{1}{2} \phi_{x}^{2}+\frac{1}{2} \phi_{t}^{2}+(1-\cos \phi)\right] d x .
$$

For the pure sine-Gordon fluxon of Eq. (6), this energy may be calculated explicitly as

$$
H_{f 0}=8 /\left(1-u^{2}\right)^{1 / 2}=P_{f 0} / u \text {. }
$$

Thus, an energy variation given by Eq. (14) may be related to a velocity variation through Eq. (16), whereupon the calculation of $X(t)$ proceeds as before. An additional approximation introduced here is that we can neglect the effects of phase shift and dissipation during reflections ${ }^{17}$ and that the energy variation of Eq. (14) occurs instantaneously.

\section{B. In-line geometry}

The analysis for the in-line geometry, described by Eqs. (3a) $-(3 c)$, is considerably simplified by the observation that

$$
\gamma=0 \text { implies } u_{\infty}=0 \text { implies } z_{\infty}=0,
$$

so that Eq. (12) is replaced by

$$
z(t)=z_{0} \exp (-\alpha t)
$$

and Eq. (13) is replaced by

$$
X(t)=x_{0}-\frac{1}{\alpha} \ln \left(\frac{z+\left(z^{2}+1\right)^{1 / 2}}{z_{0}+\left(z_{0}^{2}+1\right)^{1 / 2}}\right) .
$$

The energy variation at the boundaries, however, becomes now slightly more complicated, in that Eq. (14) is replaced by

$$
\Delta H_{f}=4 \pi(\kappa \pm \eta) \text {, }
$$

so that we now have to deal with two parameters, rather than one, as in Eq. (14).

\section{PHASE-LOCKING MAP}

Perhaps the strongest limitation to the applicability of the reduced model described earlier derives from the assumption of single fluxon dynamics. Physically, sufficiently large or sufficiently small energy exchange terms can give rise to either the creation or annihilation of fluxons; such effects are clearly manifested in computer simulations of the full PDE model. ${ }^{20}$ An ad hoc numerical artifice for treating fluxon annihilation in in-inline 
geometry junctions is described in Sec. VI later. As regards fluxon creation, we may note that a boundary term, $\eta$ or $\kappa>2$, is sufficient to inject fluxons into a junction under static conditions; this value is presumably thus an upper limit for single fluxon dynamics with time-varying boundary terms. Inasmuch as more detailed indications are not available from the reduced model itself, we will simply assume in what follows that $\eta$ and $\kappa$ have values such that creation and/or annihilation effects do not occur.

\section{A. Overlap geometry}

We take for the term $\eta$ in Eq. (2b) the form

$$
\eta=\eta_{0} \sin \left(\omega_{\mathrm{rf}} t+\theta\right)
$$

as a model of the microwave field acting upon the junction. As a mathematical convenience, we consider a periodically extended junction structure of length $L$ lying along the positive $x$ axis, between 0 and $+\infty$, in such a way that the back and forth shuttling motion of the fluxon in the physical junction is transformed into a unidirectional, left-to-right motion on the extended structure, with boundary reflection effects taking place at spatial points equal to integer multiples of $L$. A simple mathematical artifice that describes this situation is to rewrite Eq. (5) as

$$
\frac{d P_{f}}{d t}=-\alpha P_{f}+2 \pi \gamma+4 \pi \eta \sum_{k=0}^{\infty}(-1)^{k+1} \delta(X(t)-k L),
$$

in which $\delta()$ is the Dirac $\delta, X(t)$ is given by Eq. (13), and the factor $(-1)^{k+1}$ accounts for the alternating sign (for fixed $\eta$ ) of the energy variation felt by the fluxon during successive reflections.

We define the fundamental period of a fluxon oscillation to be the time employed by the fluxon to complete one back and forth round trip along the physical junction or, equivalently, to travel from $x=k L$ to $x=(k+2) L$ on the extended structure. Thus, the condition for phase locking at the fundamental frequency is, with an obvious notation

$$
T_{k, k+2}=T_{k, k+1}+T_{k+1, k+2}=\frac{2 \pi}{\omega_{\mathrm{rf}}} .
$$

In addition, we may a priori expect to observe both harmonic and subharmonic phase-locking phenomena. Thus, the general condition for phase locking is

$$
T_{k, k+2}=\frac{m}{n} \frac{2 \pi}{\omega_{\mathrm{rf}}}
$$

where $m$ and $n$ are integers.

In the absence of a microwave field, the back and forth shuttling of a fluxon in a long Josephson junction gives rise to a structure called a zero-field step in the currentvoltage characteristic of the junction. ${ }^{3}$ In terms of our model, current is proportional to the parameter $\gamma$, and voltage is proportional to the average fluxon propagation velocity $u_{\mathrm{av}}$, defined as

$$
u_{\mathrm{av}} \equiv \frac{2 L}{T_{k, k+2}} \text {. }
$$

Accordingly, phase locking of a fluxon oscillation to an external field is manifested experimentally by the appearance of a constant-voltage step in the current-voltage characteristic. In terms of our model, the height in current of such a step will be determined by how much we can vary the parameter $\gamma$ without breaking the locking condition of Eq. (24).

We can now proceed to calculate the dynamics of a fluxon in the system described by Eq. (22). Having specified the parameters $\alpha, \gamma, L, \eta_{0}, \omega_{\mathrm{rf}}$, and $\theta$, we choose an arbitrary initial value, $z_{0}^{(0)}$, of $z$ at $x=0, t=0$ [setting $x_{0}$ in Eq. (13) to zero]. By inverting (numerically) Eq. (13), i.e., by imposing $X\left(T_{0,1}\right)=L$, we calculate the time of flight, $T_{0,1}$, from $x=0$ to $x=L$. Inserting $T_{0,1}$ into Eq. (12), we calculate the final value, $z_{f}^{(1)}$, of $z$ at $x=L$, i.e., $z_{f}^{(1)}=z\left(T_{0,1}\right)$. At this point, from the energy variation, $\Delta H_{f}$ of Eq. (14), we calculate a $z$ variation, $\Delta z^{(1)}$, which, when added to $z_{f}^{(1)}$, defines a new initial value, $z_{0}^{(1)}$, of $z$ at $x=L, t=T_{0,1}$. Explicitly, we find

$$
z_{0}^{(1)}=\left[\left[\left[\left(z_{f}^{(1)}\right)^{2}+1\right]^{1 / 2}+(-1)^{k+1} \frac{\pi \eta_{0}}{2} \sin \left(\omega_{\mathrm{rf}} t+\theta\right)\right]^{2}-1\right]^{1 / 2}
$$

where, in this case, $k=0$ and $t=T_{0,1}$. We then iterate this procedure as desired for successive spatial intervals of length $L$, substituting for $t$ the sum of the preceding times of flight. In this way, Eqs. (12), (13), and (26), generalized to arbitrary $k$, constitute a discrete functional map for the quantities $T_{k, k+1}$ and $z_{0}^{(k+1)}$. Fixed points of this map correspond to phase-locked states of the fluxon dynamics, as given by Eq. (24).

\section{B. In-line geometry}

In this case, from Eq. (20), there are two parameters that contribute to the energy variation at the boundaries, viz., the bias current, $\kappa$, and the magnetic field, $\eta$. The effects of these two parameters on the fluxon dynamics are significantly different: for fixed, constant parameters, $\kappa$ gives rise to an energy increase at both boundaries, whereas $\eta$ gives rise to an increase at one boundary and a decrease at the other, exactly as for the overlap geometry. Thus, for the in-line geometry, Eq. (22) is replaced by

$$
\frac{d P_{f}}{d t}=-\alpha P_{f}+4 \pi \sum_{k=0}^{\infty}\left[\kappa+(-1)^{k+1} \eta\right] \delta(X(t)-k L),
$$


with $X(t)$ given by Eq. (19). Since, in the absence of a microwave field, an energy input is necessary in order to maintain fluxon propagation, we take for the term $\kappa$ the form

$$
\kappa=\kappa_{\mathrm{dc}}+\kappa_{\mathrm{rf}} \sin \left(\omega_{\mathrm{rf}} t+\theta\right),
$$

whereas for the term $\eta$ we take once again the form of Eq. (21). We consider two separate limiting cases; (i) electric coupling, in which $\kappa_{\text {rf }} \neq 0$ and $\eta_{0}=0$, and (ii) magnetic coupling, in which $\kappa_{\mathrm{rf}}=0$ and $\eta_{0} \neq 0$. For both cases we take $\kappa_{\mathrm{dc}} \neq 0$.

Calculation of the fluxon dynamics proceeds as before, with the added simplification that, for the in-line geometry, the times of flight, $T_{k, k+1}$, can be calculated explicitly, rather than by numerical inversion of a functional equation as in the case of the overlap geometry. Having specified the parameters $\alpha, \kappa_{\mathrm{dc}}, L, \kappa_{\mathrm{rf}}, \eta_{0}, \omega_{\mathrm{rf}}$, and $\theta$, we choose, as before, an arbitrary initial value, $z_{0}^{(0)}$, of $z$ at $x=0, t=0$ [setting $x_{0}$ in Eq. (19) to zero]. Following Levring et al. ${ }^{19}$ we now define the quantity $a(t)$ as

$$
z(t) \equiv \sinh [a(t)] .
$$

In terms of this quantity we can write, from Eq. (19), that

$$
a\left(T_{0,1}\right)=a_{0}^{(0)}-\alpha L \text {. }
$$

From Eqs. (18), (29), and (30) it follows that

$$
\sinh \left(a_{0}^{(0)}-\alpha L\right)=\sinh \left(a_{0}^{(0)}\right) \exp \left(-\alpha T_{0,1}\right),
$$

from which, in turn, we can write explicitly

$$
T_{0,1}=\frac{1}{\alpha} \ln \left(\frac{\sinh \left(a_{0}^{(0)}\right)}{\sinh \left(a_{0}^{(0)}-\alpha L\right)}\right) .
$$

The rest of the procedure is as before: we calculate $z_{f}^{(1)}$, this time using Eq. (18) instead of Eq. (12), and $z_{0}^{(1)}$, this time using Eq. (27) instead of Eq. (22). In terms of the variable $a(t)$ defined in Eq. (29), Eq. (26) is thus replaced by

$$
\cosh \left(a_{0}^{(1)}\right)=\cosh \left(a_{0}^{(0)}-\alpha L\right)+\frac{\pi}{2}\left[\kappa_{\mathrm{dc}}+K \sin \left(\omega_{\mathrm{rf}} t+\theta\right)\right],
$$

where $K=\kappa_{\mathrm{rf}}$ for electric coupling and $K=(-1)^{k+1} \eta_{0}$ for magnetic coupling. As in Eq. (26), in this case $k=0$ and $t=T_{0,1}$, whereas in general $k$ is an arbitrary integer and $t$ is the sum of the preceding times of flight.

\section{EXISTENCE AND STABILITY OF FIXED POINTS}

\section{A. In-line geometry}

The fact that the phase-locking map for the in-line geometry can be written in explicit form renders convenient the analysis of existence and stability of fixed points in this case. To this end, we note first that in the generalization of Eq. (33), the term $t$ is a sum of preceding times of flight; this means that we are dealing with a map with memory. To eliminate (formally) this fact, we define the variable

$$
S_{n} \equiv \sum_{k=0}^{n-1} T_{k, k+1}
$$

Secondly, we note that $S_{n}$ appears in the map only in the argument of the sine function. This means that we may consider $S_{n}$ to be defined modulo $2 \pi / \omega_{\text {rf }}$, which implies that we are working in a cylindrical phase space. Finally, for convenience, we define the variable

$$
U_{n} \equiv \cosh \left(a_{0}^{(n)}\right)
$$

which, from Eqs. (11) and (29), is seen to be just the Lorentz contraction factor at the $n$th boundary. In terms of these variables, the in-line map may be written explicitly as

$$
\begin{aligned}
& S_{n+1}=S_{n}+\frac{1}{\alpha} \ln \left(\frac{\left(U_{n}^{2}-1\right)^{1 / 2}}{\cosh (\alpha L)\left(U_{n}^{2}-1\right)^{1 / 2}-\sinh (\alpha L) U_{n}}\right) \bmod \frac{2 \pi}{\omega_{\mathrm{rf}}} \\
& U_{n+1}=\cosh (\alpha L) U_{n}-\sinh (\alpha L)\left(U_{n}^{2}-1\right)^{1 / 2}+\frac{\pi}{2}\left[\kappa_{\mathrm{dc}}+K \sin \left(\omega_{\mathrm{rf}} S_{n+1}+\theta\right)\right] .
\end{aligned}
$$

From the definition of the term $K\left[K=\kappa_{\mathrm{rf}}\right.$ or $\left.K=(-1)^{n+1} \eta_{0}\right]$, it is clear that the symmetry of the map is different for electric and for magnetic coupling. In particular, in the case of magnetic coupling, we expect to find fixed points for identical times of flight, $T_{k, k+1}$ for all $k$, at the fundamental frequency and all odd subharmonics, viz.,

$$
T_{k, k+1}=m \pi / \omega_{\mathrm{rf}}, \quad m=1,3,5, \ldots .
$$

This means that

$$
\omega_{\mathrm{rf}} S_{n+1}=(n+1) \pi(\bmod 2 \pi) .
$$

For even subharmonics, equal times of fight are not, in general, possible. For electric coupling, the situation is reversed: equal times of flight are possible for even subharmonics, i.e., $m=2,4,6, \ldots$, but not for the fundamental frequency or for odd subharmonics. In this case, $\omega_{\mathrm{rr}} S_{n+1}=0(\bmod 2 \pi)$ at the fixed point.

To calculate the fixed points of Eqs. (36), which we denote $S^{*}, U^{*}$, for equal times of flight, we use the fact that, at the fixed point, $U_{n+1}=U_{n}$ and

$$
S_{n+1}-S_{n}=T_{n, n+1}=m \pi / \omega_{\mathrm{rf}} .
$$

Here, odd values of $m$ apply to magnetic coupling and even values to electric coupling. Inserting these facts into Eqs. (36), we find that 


$$
\begin{aligned}
S^{*} & =\frac{\arcsin \left[\left(\bar{K}-\kappa_{\mathrm{dc}}\right) /|K|\right]-\theta}{\omega_{\mathrm{rf}}}, \\
U^{*} & =\frac{\cosh (\alpha L)-E}{\left[1-2 E \cosh (\alpha L)+E^{2}\right]^{1 / 2}},
\end{aligned}
$$

with

$$
E \equiv \exp \left(-m \pi \alpha / \omega_{\mathrm{rf}}\right)
$$

and

$$
\bar{K} \equiv \frac{2[\cosh (\alpha L)-1](1+E)}{\pi\left[1-2 E \cosh (\alpha L)+E^{2}\right]^{1 / 2}} .
$$

By using $|K|$, i.e., absolute value of $K$, in Eq. (37a) we obtain that Eqs. (37) apply to both magnetic and to electric coupling. From Eqs. (36b) and (37a) it is apparent that $\bar{K}$ is just the value of the bias current $\kappa_{\mathrm{dc}}$ at which the rfinduced step intersects the unperturbed zero-field step: For $\kappa_{\mathrm{dc}}=\bar{K}$, setting $K=0$ yields the same point as that obtained by setting $\theta=0$ with $K \neq 0$.

From Eq. (37b), a necessary condition for the existence of $U^{*}$ is that the argument of the square root be positive, which implies that $L<m \pi / \omega_{\mathrm{rf}}$. Physically, this condition says only that the limiting maximum fluxon velocity is 1 . Assuming this condition to be satisfied, we conclude from Eq. (37a) that $S^{*}$, and hence the fixed point, exists for bias currents in the range

$$
\overline{\boldsymbol{K}}-|\boldsymbol{K}|<\kappa_{\mathrm{dc}}<\overline{\boldsymbol{K}}+|\boldsymbol{K}| \text {. }
$$

We note that this result is in agreement with that reported by Chang. ${ }^{12}$

To analyze the stability of this fixed point we linearize the map of Eqs. (36) around the fixed point of Eqs. (37), i.e., we consider the variational equation

$$
\left[\begin{array}{l}
\delta S_{n+1} \\
\delta U_{n+1}
\end{array}\right]=\underline{J}\left(S^{*}, U^{*}\right)\left(\begin{array}{l}
\delta S_{n} \\
\delta U_{n}
\end{array}\right),
$$

where $\underline{J}\left(S^{*}, U^{*}\right)$ denotes the Jacobian matrix of Eqs. (36) evaluated at the fixed point, and $\delta S$ and $\delta U$ denote small deviations from this point. The condition for stability is that the eigenvalues of $\underline{J}\left(S^{*}, U^{*}\right)$ lie within the unit circle in the complex plane. After somewhat lengthy calculations, we find that this condition reduces to

$$
0<\cos \left(\omega_{\mathrm{rr}} S^{*}+\theta\right)<\Lambda /|K|,
$$

with

$$
\Lambda \equiv \frac{4 \alpha E(1+E) \sinh ^{2}(\alpha L)}{\pi \omega_{\mathrm{rf}}\left[1-2 E \cosh (\alpha L)+E^{2}\right]^{3 / 2}} .
$$

Recalling that $\omega_{\mathrm{rr}} S^{*}=0(\bmod 2 \pi)$ for electric coupling and $\omega_{\mathrm{rf}} S^{*}=0$ or $\pi(\bmod 2 \pi)$ for magnetic coupling, we may simplify condition (41) to

$$
0<\cos \theta<\Lambda /|K| \text {. }
$$

The left-hand inequality in condition (43) says that, for stability, the fluxon must arrive at the junction boundary at which the energy variation is positive during the positive-slope edge of the microwave field. For $\Lambda>|K|$, the right-hand inequality says that the range of stability is the entire range of existence, as given by condition (39). For $\Lambda \leq|K|$, we may cast the stability condition into a more useful form by substituting for $S^{*}$ in condition (41) the value given by Eq. (37a). This procedure yields that the fixed point is stable for bias currents in the ranges

$$
\begin{aligned}
& \bar{K}-|K|<\kappa_{\mathrm{dc}}<\bar{K}-\left(|K|^{2}-\Lambda^{2}\right)^{1 / 2}, \\
& \bar{K}+\left(|K|^{2}-\Lambda^{2}\right)^{1 / 2}<\kappa_{\mathrm{dc}}<\bar{K}+|K| .
\end{aligned}
$$

We emphasize that our analysis predicts the onset of instability for the equal-times-of-flight fixed point first at the center of the step, inasmuch as $\cos \theta=1$ implies that $\kappa_{\mathrm{dc}}=\bar{K}$, rather than at the extremes of the step, as one might intuitively expect. Moreover, from Eqs. (38a) and (42), we note that $\Lambda$ decreases rapidly with increasing $m$, which means that the stability range for subharmonics decreases rapidly with increasing subharmonic order.

The question now arises: what happens when the equal-times-of-flight solution loses stability? In an attempt to answer this question, we hypothesize the existence of a solution having

$$
\omega_{\mathrm{rf}} T_{n, n+1}=m \pi-\Delta
$$

and

$$
\omega_{\mathrm{rf}} T_{n+1, n+2}=m \pi+\Delta,
$$

where, again, odd values of $m$ apply to magnetic coupling and even values to electric coupling. Physically, such a solution leaves the junction biased on the same rf-induced step, but now a new half-harmonic frequency appears in the spectrum.

A solution of the type specified by Eqs. (45) corresponds to a fixed point of the second iterate of the map of Eqs. (36), i.e., it has

$$
S_{n+2}=S_{n}\left(\bmod 2 \pi / \omega_{\mathrm{rf}}\right)
$$

and $U_{n+2}=U_{n}$. This means that

$$
\omega_{\mathrm{rP}} S_{n+1}=(n+1) m \pi-\Delta,
$$

and

$$
\omega_{r f} S_{n+2}=0(\bmod 2 \pi) .
$$

Inserting this information into Eqs. (36) and defining the quantity

$$
D \equiv \exp \left(\alpha \Delta / \omega_{\mathrm{rf}}\right),
$$

we find the pair of equations:

$$
\begin{aligned}
& \frac{\pi}{2}\left[x_{\mathrm{dc}}+|K| \sin (\theta-\Delta)\right]=\frac{\cosh (\alpha L)-(E / D)}{\left[1-2(E / D) \cosh (\alpha L)+(E / D)^{2}\right]^{1 / 2}}-\frac{1-E D \cosh (\alpha L)}{\left[1-2 E D \cosh (\alpha L)+(E D)^{2}\right]^{1 / 2}}, \\
& \frac{\pi}{2}\left(\kappa_{\mathrm{dc}}+|K| \sin \theta\right)=\frac{\cosh (\alpha L)-E D}{\left[1-2 E D \cosh (\alpha L)+(E D)^{2}\right]^{1 / 2}}-\frac{1-(E / D) \cosh (\alpha L)}{\left[1-2(E / D) \cosh (\alpha L)+(E / D)^{2}\right]^{1 / 2}}
\end{aligned}
$$


Eqs. (47) can be solved for $\theta$ and $\Delta$. To simplify this calculation, we now assume that the equal-times-of-flight solution bifurcates smoothly into the two-times-of-flight solution, and we study this solution near the point of bifurcation, i.e., we assume that $|\Delta| \ll 1$, and we linearize Eqs. (47). Under this assumption, we find that

$$
\begin{aligned}
& \cos \theta=\Lambda /|K|, \\
& \sin \theta=\frac{\bar{K}-\kappa_{\mathrm{dc}}}{|K|}+\frac{\Delta}{2} \cos \theta,
\end{aligned}
$$

Comparing Eq. (48a) and condition (43), we see that the two-times-of-flight solution comes into existence at just the point where the equal-times-of-flight solution loses stability. An obvious condition for its existence is $\cos \theta \leq 1$. We have not attempted to analyze the stability of this second-iterate fixed point. Instead, we refer to Sec. VI below for numerical results regarding multipletimes-of-flight solutions.

We now consider the case of even subharmonics for magnetic coupling and odd subharmonics (including the fundamental) for electric coupling. As mentioned earlier, the symmetry of Eqs. (36) does not, in general, permit equal-times-of-flight solutions in these cases. We therefore hypothesize once again the existence of a solution of the type specified by Eqs. (45), where, however, even values of $m$ now apply to magnetic coupling and odd values to electric coupling. With this change, we see that such solutions are again described by Eqs. (47), provided that we change the plus sign preceding the term $|K|$ in Eq. (47a) [but not in Eq. (47b)] to a minus sign. For simplicity, we again assume that $|\Delta| \ll<1$, and we expand the modified Eqs. (47) to the second order. The result is

$$
\begin{aligned}
& \sin \theta=\frac{\bar{K}-\kappa_{\mathrm{dc}}}{|K|}+\frac{\Lambda}{2|K|} \Delta+\frac{\Lambda \Gamma}{4|K|} \Delta^{2}, \\
& \Delta=\frac{2|K|}{\Lambda} \sin \theta\left(1-\frac{|K|}{\Lambda} \cos \theta\right),
\end{aligned}
$$

with

$$
\Gamma \equiv \frac{\alpha}{\omega_{\mathrm{rf}}}+\frac{3 \Lambda}{2 \bar{K}} \tanh ^{2}\left(\frac{\alpha L}{2}\right) .
$$

We have assumed that $|\Delta| \ll 1$. From Eq. (49b), we see that, for consistency, we must have $|K|<<\Lambda / 2$, which thus gives an explicit condition for checking the validity of the approximation. Combining Eqs. (49a) and (49b), we find that the extremes of the step occur for $\tan (2 \theta)=1 / \Gamma$. Using this fact, we conclude that the fixed point exists for bias currents in the range

$$
\begin{aligned}
& \bar{K}+\frac{|K|^{2}}{2 \Lambda}\left[\Gamma-\left(1+\Gamma^{2}\right)^{1 / 2}\right] \\
&<\kappa_{\mathrm{dc}}<\bar{K}+\frac{|K|^{2}}{2 \Lambda}\left[\Gamma+\left(1+\Gamma^{2}\right)^{1 / 2}\right] .
\end{aligned}
$$

The height in current of these steps should thus vary linearly with the applied microwave power (in the lowpower limit), rather than as the square root of the power as for the equal-times-of-flight steps described by condi- tion (39). However, in contrast with the equal-times-offlight steps, the centers of these steps do not intersect the unperturbed characteristic, but they are shifted upward in current by an amount proportional to the applied microwave power. We note that these conclusions are in agreement with those reported by Chang. ${ }^{12}$

\section{B. Overlap geometry}

Analysis of the existence and stability of fixed points for the overlap geometry is complicated by the fact that the phase-locking map is available only in implicit form. Qualitatively, since there can be only magnetic coupling in this case, we are led to expect again the existence of equal-times-of-flight solutions for the fundamental frequency and all odd subharmonics. Assuming this to be true, we can, using the ideas developed in Ref. 16, calculate approximately, in the limit of sufficiently small values of $\eta_{0}$, the range of bias current for the existence of the fixed point. To this end, we note that, in the absence of a microwave field, the average fluxon propagation velocity, given by Eq. (25), is just $u_{\infty}$. Equation (10) therefore gives the current-voltage characteristic of the unperturbed first zero-field step. From Eq. (22), which describes the force acting on the fluxon, we see that, under the assumption that $T_{k, k+1}=m \pi / \omega_{\mathrm{rf}}$, with $m$ odd, the total work performed on the fluxon in propagating from $x=k L$ to $x=(k+2) L$ is just $4 \pi \gamma L+8 \pi \eta$. For constant work, we expect $u_{\mathrm{av}}$ to remain (approximately) constant. ${ }^{17}$ This implies that the average junction voltage remains constant, which means that the junction is based on an rf-induced step. Since $\eta$ can vary in the range $\pm \eta_{0}$, we conclude that the fixed point exists for bias currents in the range

$$
\bar{\gamma}-2 \eta_{0} / L<\gamma<\bar{\gamma}+2 \eta_{0} / L
$$

where

$$
\bar{\gamma} \equiv \frac{4 \alpha L \omega_{\mathrm{rf}}}{\pi\left[(m \pi)^{2}-\left(\omega_{\mathrm{rf}} L\right)^{2}\right]^{1 / 2}},
$$

which, as for the in-line geometry, is just the current value at which the rf-induced step intersects the unperturbed zero-field step.

\section{NUMERICAL RESULTS}

\section{A. Computational procedure}

The basic idea of the computational procedure has been outlined briefly in Sec. IV earlier. Here, we fill in some salient operational details. To calculate the current-voltage characteristic of the junction we proceed as follows:

(i) Fix the parameters of the map. These are $\alpha, \gamma, L$, $\eta_{0}, \omega_{\mathrm{rf}}$, and $\theta$ for the overlap geometry and $\alpha, \kappa_{\mathrm{dc}}, L, x_{\mathrm{rf}}$ (for electric coupling), $\eta_{0}$ (for magnetic coupling), $\omega_{\mathrm{rf}}$ and $\theta$ for the in-line geometry.

(ii) Iterate the map a certain number, say $M$, times to eliminate transients.

(iii) Iterate the map another $N$ times to calculate the 
average time of flight from $T_{\mathrm{av}}=\left(t_{M+N}-t_{M}\right) / N$. For multiple-times-of-flight solutions, measure the individual times of flight in a similar fashion.

(iv) Double $M$ and $N$ until $T_{\text {av }}$, as well as individual times of flight, if present, converge to within a designated uncertainty (typically $1 \%$ ).

(v) Calculate the average voltage from $V_{\mathrm{av}}=2 \pi / T_{\mathrm{av}}$. In a phase-locked state $V_{\mathrm{av}}=2 \omega_{\mathrm{rf}} / m$, where $m$ is the subharmonic number.

(vi) Plot $\gamma$ versus $V_{\text {av }}$ for the overlap geometry or $\kappa_{d c}$ versus $V_{\text {av }}$ for the in-line geometry. To check for the presence of hysteresis (see further), it is necessary both to increase and decrease monotonically $\gamma$ or $x_{\mathrm{dc}}$.

Since the map is available only implicitly for the overlap geometry, it is necessary to invert Eq. (13) numerically to obtain the successive times of flight, $T_{k, k+1}$. Because the time dependence of $X(t)$ in Eq. (13) is very smooth and regular, we have found a simple Newton method to be adequate for this purpose.

For the in-line map, it can occur that the fluxon does not acquire enough energy to cross the junction. This fact is evident from Eq. (32): the time of flight $T_{k, k+1}$ is defined only if $a_{0}^{(k)}>\alpha L$. This is purely an artifact of the map, which is not present in the PDE system of Eqs. (3). It is because of the fact, apparent from Eq. (27), that in the map the fluxon receives energy only at the mathematical boundaries of the junction, whereas in the PDE the fluxon "feels" the effect of the boundary conditions of Eqs. (3b) and (3c) also in the interior of the junction. To obviate this limitation of the map we have employed the following ad hoc artifice: If we find a given $a_{0}^{(k)}$ to be less than $\alpha L$, we arbitrarily set the corresponding $T_{k, k+1}$ to a large, but finite, value and continue with the iterations. We then double $M$ and repeat the run, using as initial values for $z_{0}^{(0)}$ and $\theta$ the final values obtained in the previous run. We repeat this procedure until either the solution settles into a steady state that does not require such resets or the value required for $M$ exceeds 1000 . In the former case we proceed with the calculation of $T_{\mathrm{av}}$, etc. We interpret the latter case to mean that the junction switches to the zero-voltage state.

An aspect of fluxon oscillators that is of considerable interest to the experimentalist is the frequency spectrum of the radiation emitted by the oscillator. To model qualitatively this aspect of fluxon dynamics we represent the time sequence $T_{k, k+2}$ (we use $T_{k, k+2}$ rather than $T_{k, k+1}$ to reflect the fact that we are "detecting" radiation from just one end of the junction) as a sequence of unit $\delta$ functions, and we calculate the Fourier transform of this sequence.

\section{B. In-line geometry}

Figure 1 shows two examples of the current-voltage characteristic of an in-line junction with magnetic coupling; Fig. 1(a) refers to locking at the fundamental frequency $(m=1)$ and Fig. 1(b) to locking at the third subharmonic $(m=3)$. In order to directly compare the two results we have set $\omega_{\text {rf }}$ in Fig. $1(b)$ to three times the value used in Fig. 1(a). In both Fig. 1(a) and Fig. 1(b) the smooth curve is the profile of the zero-field step in the ab- sence of a microwave field, and the discontinuous curve is the step induced by a field of amplitude $\eta_{0}=0.4$. From condition (39) the bias range for the existence of both steps is identical, equal to $\pm \eta_{0}$ about the center point. From condition (43) the equal-times-of-flight fixed point corresponding to Fig. 1(a) should be stable for $\eta_{0} \leq 4.824$; that corresponding to Fig. 1(b) should be stable for $\eta_{0} \leq 1.608$. Both of these limits are considerably larger than the value used. The two characteristics are almost identical, the most salient difference being that the lower portion of the step in Fig. 1(b) is slightly shorter than that in Fig. 1(a). This difference is attributable to the mechanism, described earlier, of switching to zero voltage, which is not contained in the theoretical analysis.

Figure 2 indicates the situation for locking at the second subharmonic $(m=2)$. Figure 2(a) refers to electric
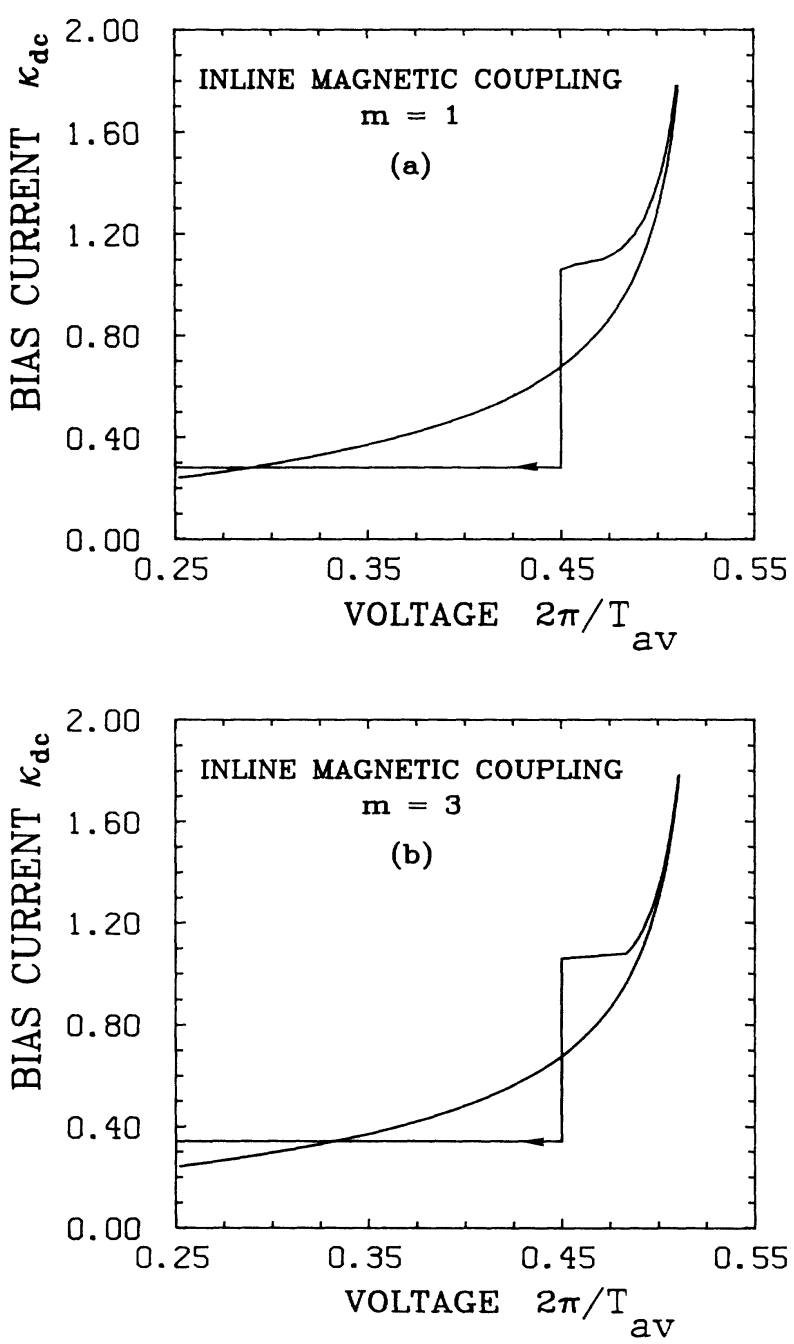

FIG. 1. Current-voltage characteristic of in-line junction with magnetic coupling. Smooth curve: no field; discontinuous curve: with field. (a) Fundamental frequency, $m=1, \omega_{\mathrm{rf}}=0.225$; (b) third subharmonic, $m=3, \omega_{\mathrm{rf}}=0.675$. Parameters: $\alpha=0.05$, $L=12, \eta_{0}=0.4, M=100$, and $N=200$. The arrows represent the switching to the zero-voltage state because of the fluxon annihilation. 

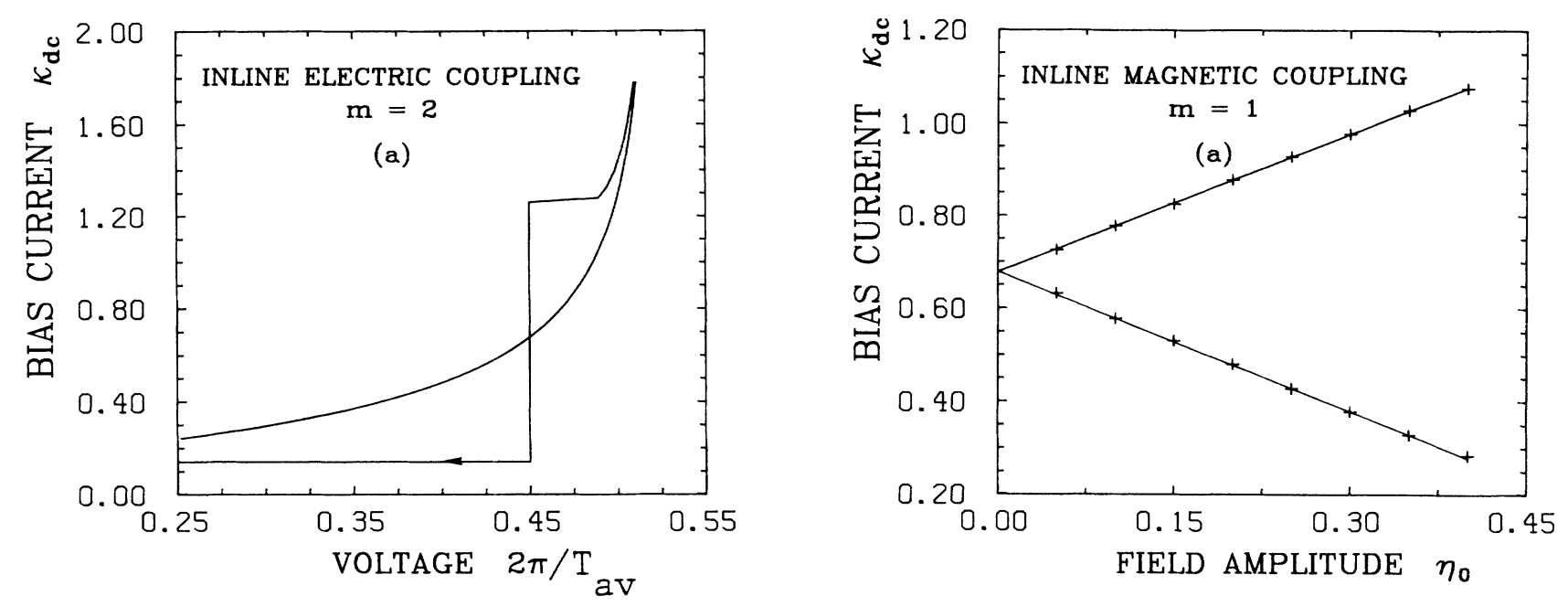

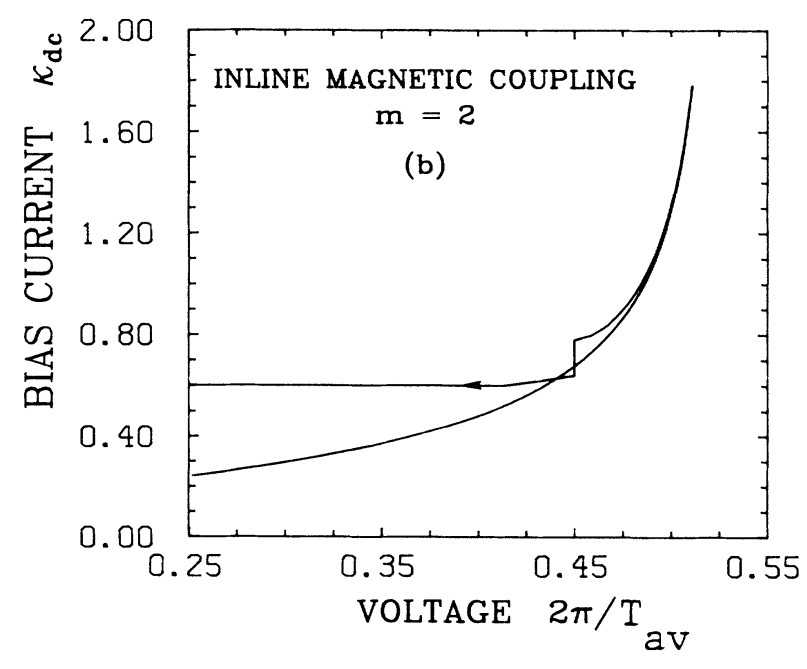

FIG. 2. Current-voltage characteristic of in-line junction at second subharmonic. Smooth curve: no field; discontinuous curve: with field. (a) Electric coupling; (b) magnetic coupling. Parameters: $\alpha=0.05, L=12, \kappa_{\mathrm{rf}}=\eta_{0}=0.6, \omega_{\mathrm{rf}}=0.450, M=100$, and $N=200$.

coupling, again governed by conditions (39) and (43), and Fig. 2(b) to magnetic coupling, described by condition (51). The qualitative difference between the two cases is evident.

Figure 3 demonstrates the dependence of the locking range in bias current on the field amplitude. Figure 3(a) refers to the situation of Fig. 1(a) and Fig. 3(b) to that of Fig. 2(b). In Fig. 3 the solid curves are the analytical predictions, given by condition (39) for Fig. 3(a) and by condition (51) for Fig. 3(b), and the crosses are the extremes of the step obtained by numerical solution of the map. The agreement is quite good in both cases. We have verified that the crosses in Fig. 3(b) correspond to twotimes-of-flight solutions. Moreover, from Eq. (42), $\Lambda=2.41$ for the parameter values used, so the condition that $\eta_{0} \ll \Lambda / 2$ is amply satisfied, but we have not attempted to perform a stability analysis for multipletimes-of-flight solutions.

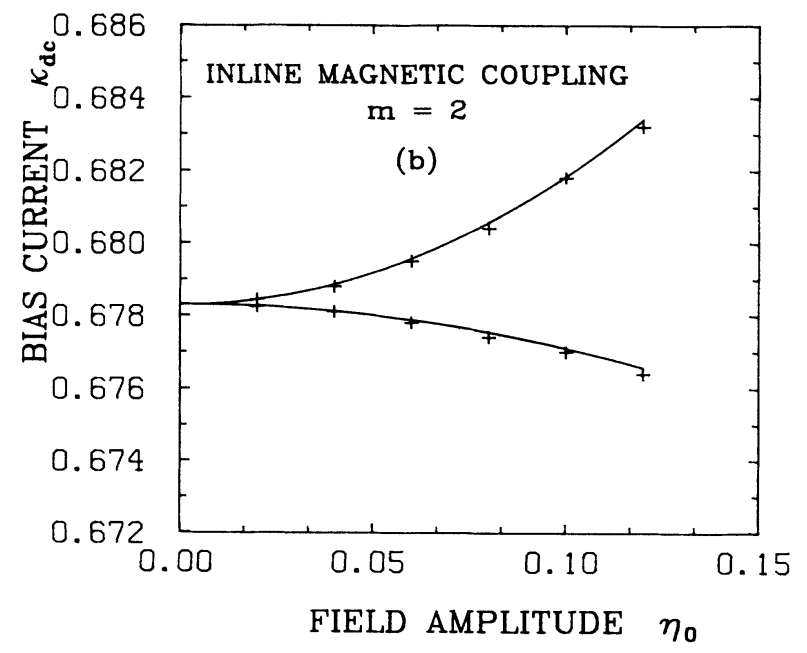

FIG. 3. Dependence of locking range in bias current on field amplitude for in-line junction with magnetic coupling. (a) Fundamental frequency, $m=1, \omega_{\mathrm{rf}}=0.225$. Solid curve: Eq. (39); crosses: numerical result. (b) Second subharmonic, $m=2$, $\omega_{\text {rf }}=0.450$. Solid curve: Eq. (51); crosses: numerical result. Parameters: $\alpha=0.05, L=12 . \quad M$ and $N$ are different for different field values.

Figure 4 illustrates the evolution of multiple-times-offlight solutions for in-line junctions with magnetic coupling at the third subharmonic. Equation (48a) predicts that, for the parameter values used, $\cos \theta=1$ for $\eta_{0}=0.03324$. The numerical result for the first bifurcation in Fig. 4 agrees with this prediction to three significant digits. In addition, Fig. 4 shows a second bifurcation at $\eta_{0}=0.0403$ and a third at $\eta_{0}=0.0419$. The appearance of Fig. 4 strongly suggests the existence of a Feigenbaum cascade. ${ }^{21}$ Assuming this to be the case, we find from these first three bifurcations an estimate of 4.44 for the Feigenbaum ratio, as compared with the universal asymptotic value of 4.669 . . .

Figure 5 shows an example of superharmonic locking. The figure refers to an in-line junction with electric coupling, having $m=1$ and $n=2$ in Eq. (24) (we have tacitly 


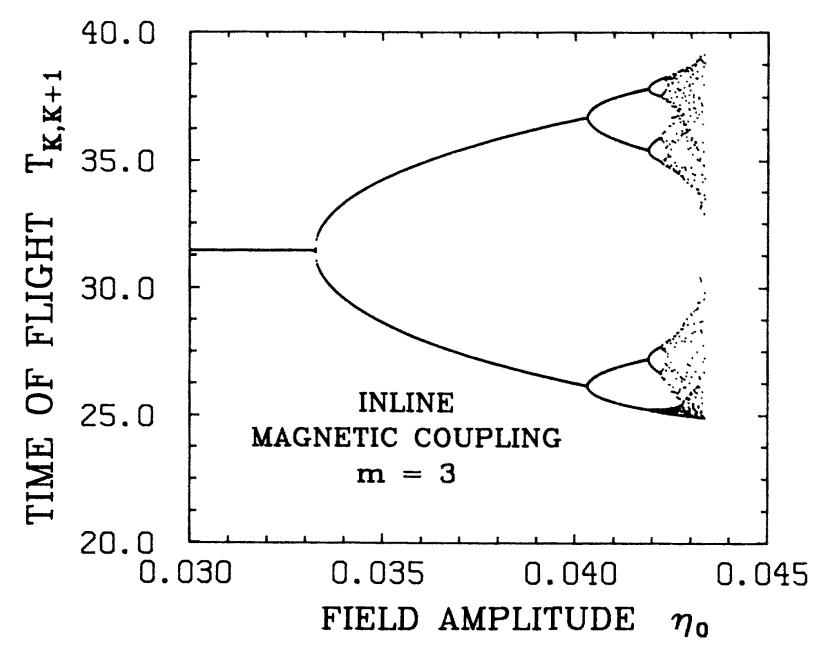

FIG. 4. Dependence of time of flight on field amplitude for in-line junction with magnetic coupling at third subharmonic. Parameters: $\alpha=0.05, \kappa_{\mathrm{dc}}=0.1295, L=10$, and $\omega_{\mathrm{rf}}=0.3$. $M$ and $N$ are different for different field values.

assumed $n=1$ in all of the preceding discussion). The right-hand curve in Fig. 5 is the profile of the zero-field step in the absence of a microwave field, and the left-hand one is the step induced by a field of amplitude $\kappa_{\mathrm{rf}}=0.8$. The induced step corresponds to a four-times-of-flight solution. We have not yet studied the case of superharmonic locking in any significant detail, but a few preliminary calculations consistently show the following distinctive features: (i) The induced steps are very small. (ii) They are shifted upward in current with respect to the unperturbed characteristic. These results are qualitatively consistent with the experimental results of Scheuermann et al. ${ }^{9}$ and with the analysis of Chang. ${ }^{12}$

As mentioned earlier, the field-induced steps calculated

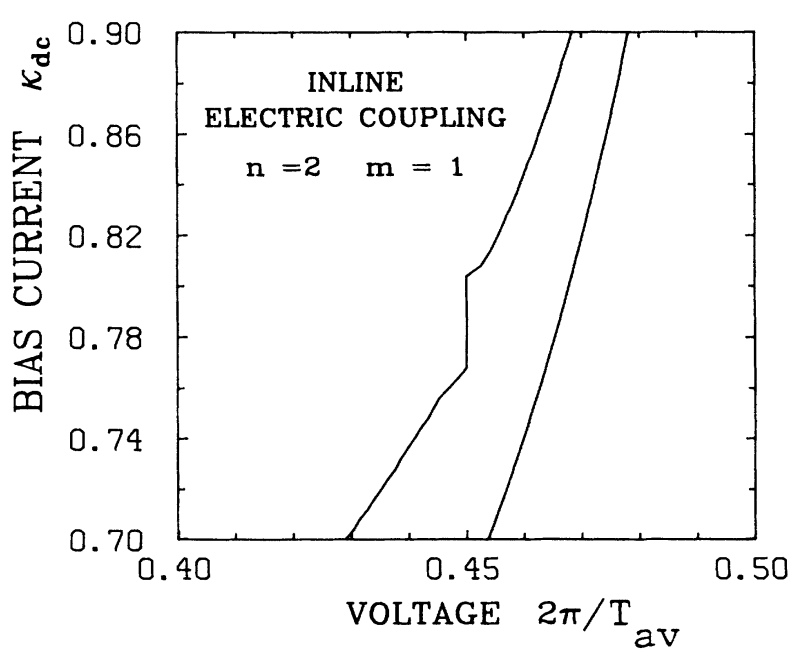

FIG. 5. Current-voltage characteristic of in-line junction with electric coupling at second superharmonic, $n=2, m=1$. Right-hand curve: no field; left-hand curve: with field. Parameters: $\alpha=0.05, L=12, \kappa_{\mathrm{rf}}=0.8, \omega_{\mathrm{rf}}=0.1125, \quad M=300$, and $N=300$. from the map sometimes show hysteretic behavior, in qualitative agreement with experimental observations on real junctions. ${ }^{22}$ We have not yet conducted an exhaustive study of this phenomenon, but preliminary observations suggest that: (i) Hysteresis is more readily observable with magnetic coupling than with electric coupling. (ii) Hysteresis increases with decreasing loss coefficient $\alpha$. (iii) Hysteresis increases with decreasing junction length $L$. (iv) For fixed $\alpha$ and $L$, the ratio of hysteresis current to total step height is approximately independent of field amplitude.

Figure 6 indicates the $\alpha$ dependence of hysteretic behavior for an in-line junction with magnetic coupling. A significant feature visible in Fig. 6 is that the fieldinduced step crosses the zero-current axis, extending to negative current values. This phenomenon is frequently observed experimentally. ${ }^{22}$ Zero-crossing steps have attracted interest for Josephson voltage-standard applications. $^{23}$
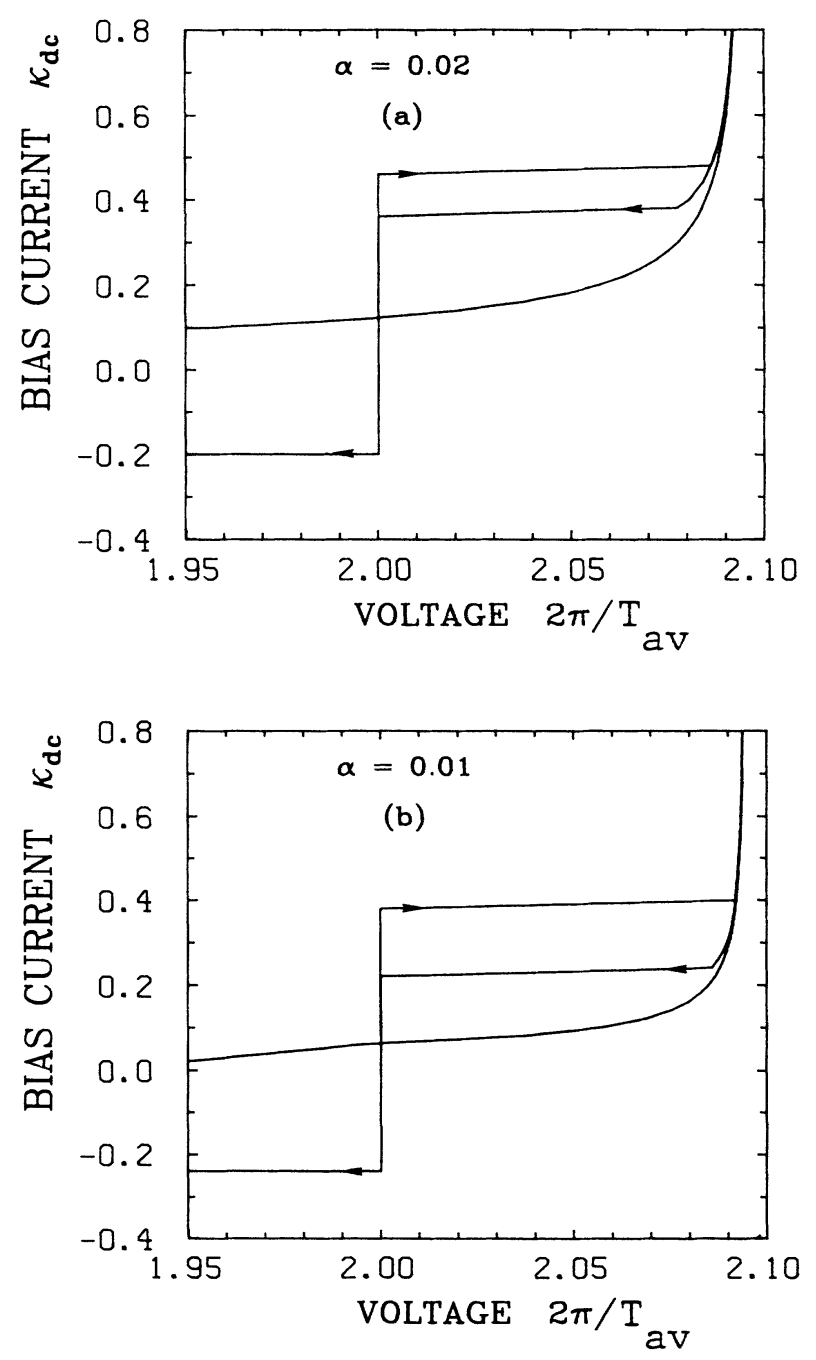

FIG. 6. Variation of hysteresis with loss parameter for in-line junction with magnetic coupling at fundamental frequency. (a) $\alpha=0.02$; (b) $\alpha=0.01$. Parameters: $L=3, \eta_{0}=0.4, \omega_{\mathrm{rf}}=1.0$, $M=100$, and $N=200$. The arrows represent switching to and from the locked state. 


\section{Overlap geometry}

The results for the overlap geometry are qualitatively very similar to those for the in-line geometry with magnetic coupling. This fact is illustrated in Fig. 7. Figures $7(\mathrm{a}), 7(\mathrm{~b})$, and $7(\mathrm{c})$ show phase locking in the same circumstances as depicted, respectively, in Figs. 1(a), 2(b), and $1(\mathrm{~b})$. Figure 7 (d), which shows the dependence of the locking range in bias current on field amplitude, may be compared with Fig. 3(a). The solid curve in Fig. 7(d) is the prediction of Eq. (52). Although this equation is only an approximation, the agreement with the numerical result (crosses) is surprisingly good.

Figure 8 shows the dynamics of the phase-locking process in the frequency domain. In this figure the fluxon frequency is defined as $2 \pi / T_{k, k+2}$, i.e., we consider locking at the fundamental frequency. The drive frequency $\omega_{\text {rf }}$ is 0.230 in the lowest trace; it increases in increments of 0.003 in the higher traces. Clearly evident in Fig. 8 is the fact that as the drive frequency approaches the unlocked fluxon frequency, frequency pulling is observed, and mixing products (the small peaks near the edges of the figure) begin to appear. At a certain point, the fluxon frequency is pulled into synchronism with the driver, where it remains locked for a certain interval (the central region of the figure). Beyond this region the fluxon frequency unlocks, and mixing products are once again seen. This behavior should be compared with Fig. 3 of Cirillo and Lloyd ${ }^{10}$ and Fig. 3 of Monaco et al. ${ }^{1}$ which depict experimental recordings of the same scenario.

\section{REFINEMENTS OF THE MODEL}

In Secs. III and IV earlier, we have made several simplifying assumptions in order to construct the phaselocking map. We now consider possible modifications of the proposed map, which take into account the following effects:
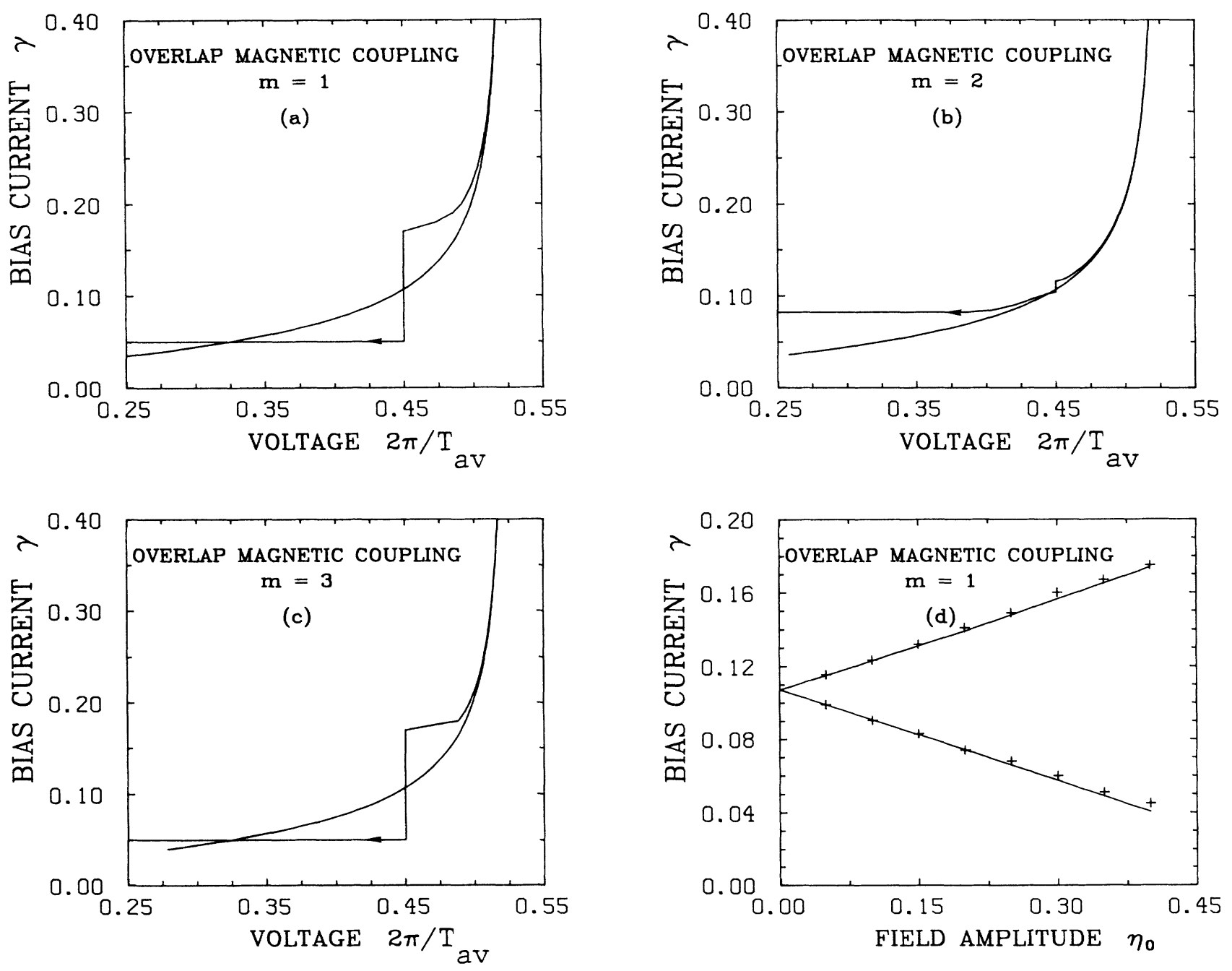

FIG. 7. Current-voltage characteristics $[(a)-(c)]$ and locking range $[(d)]$ for overlap junction with magnetic coupling. (a) $m=1$, $\eta_{0}=0.4, \omega_{\mathrm{rf}}=0.225$; (b) $m=2, \eta_{0}=0.4, \omega_{\mathrm{rf}}=0.450$; (c) $m=3, \eta_{0}=0.4, \omega_{\mathrm{rf}}=0.675$; (d) $m=1, \omega_{\mathrm{rf}}=0.225$; solid curve: Eq. (49); crosses: numerical result. Parameters: $\alpha=0.05, L=12$. The arrows represent the switching to the zero-voltage state because of the fluxon annihilation. 
(a) The presence of a constant bias current in the overlap geometry modifies the fluxon ground state, and the presence of an $\alpha$-loss term modifies the Lorentz contraction factor of the fluxon waveform.

(b) The reflection of a fluxon as an antifluxon (or vice versa) at a junction boundary introduces, even for the unperturbed sine-Gordon system, a phase shift in the fluxon position. This phase shift causes an effective advance in the fluxon motion that affects the phase-locking mechanism.

(c) During a fluxon-antifluxon reflection in the presence of an $\alpha$-loss term, a fraction of the fluxon energy is dissipated, resulting in a slowing down of the fluxon motion.

\section{A. Bias and Lorentz contraction effects for the overlap geometry}

The McLaughlin-Scott perturbative approach, ${ }^{11} \mathrm{em}$ ployed earlier, is based on the idea that if $\alpha$ and $\gamma$ in Eq. (2a) are small enough, the perturbed fluxon is adequately described by Eq. (8). In practice, however, the bias term $\gamma$ is not always a small parameter; in such cases, the true fluxon waveform is significantly different from the ansatz of Eq. (8). Ferrigno and $\mathrm{Pace}^{24}$ proposed a modified ansatz for the perturbed fluxon, given by

$$
\phi_{P}=\arcsin \gamma+4 \arctan \left(\exp \left\{-[x-X(t)]\left(1-\gamma^{2}\right)^{1 / 4} /\left[1-u^{2}(t)\right]^{1 / 2}\right\}\right),
$$

in which $X(t)$ is again given by Eq. (9). Equation (54) evidently reduces to Eq. (8) for $\gamma<<1$. The term $\arcsin \gamma$ in Eq. (54) is the shift of the fluxon ground state due to the constant bias current. Its presence gives rise to a constant energy density, and hence an infinite energy contribution for an infinite-length junction, which, however, may be removed by a simple renormalization. Although McLaughlin and Scott ${ }^{11}$ did not explicitly include this term in their ansatz, it is fairly evident that they tacitly assumed its existence. As shown in Ref. 24, the term $\left(1-\gamma^{2}\right)^{1 / 4}$ in Eq. (54) gives a nonzero limiting width for the fluxon in the limit $\gamma \rightarrow 1, u \rightarrow 1$, in (better) agreement with analytic and numerical results.

It is easy to show that the use of the ansatz of Eq. (54) in place of that of Eq. (8) in Eq. (4) and in the renormalized Eq. (15), leads to the following modifications of Eqs. (7) and (16):

$$
\hat{P}_{f 0}=P_{f 0}\left(1-\gamma^{2}\right)^{1 / 4}
$$

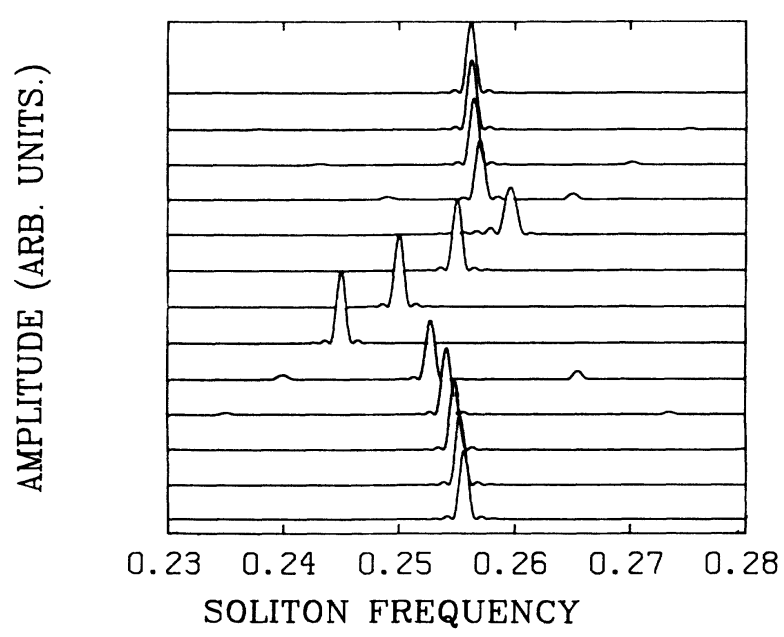

FIG. 8. Frequency-domain representation of phase-locking process for overlap junction with magnetic coupling at fundamental frequency. See text for details. Parameters: $\alpha=0.05$, $\gamma=0.34, L=12$, and $\eta_{0}=1.0$.

$$
\hat{H}_{f 0}=H_{f 0}\left(1-\gamma^{2}\right)^{1 / 4} .
$$

It is thus apparent that all of the consequences of using Eq. (54) in place of Eq. (8) can be accounted for simply by defining an effective bias current $\gamma_{\text {eff }}$, given by

$$
\gamma_{\text {eff }} \equiv \gamma /\left(1-\gamma^{2}\right)^{1 / 4} \text {, }
$$

since the substitution of $\gamma_{\text {eff }}$ for $\gamma$ in Eq. (22) leaves that equation unchanged.

\section{B. Phase-shift effects}

It is well known that the reflection of a pure sineGordon soliton from an openended boundary, i.e., a $\phi_{x}=0$ boundary condition, is mathematically equivalent to a soliton-antisoliton collision. Comparison of the asymptotic forms of the single soliton of Eq. (6) and the soliton-antisoliton doublet ${ }^{11}$ shows that such a reflection gives rise to an asymptotic spatial phase advance $\psi$ for the single soliton, given by

$$
\psi=-2\left(1-u^{2}\right)^{1 / 2} \ln u,
$$

where $u$ is the soliton propagation velocity, as in Eq. (6). Olsen et al. ${ }^{17}$ accordingly suggested that the effect of phase shift on a finite-length junction could be taken into account by defining an effective junction length $L_{\text {eff }}$, given by

$$
L_{\text {eff }}=L-\psi \text {. }
$$

For the perturbed sine-Gordon model used in the map, the result of Eq. (58) no longer holds exactly because of: (i) the presence of the perturbing terms, $\alpha$ and $\gamma$, in the PDE (ii) the presence of nonhomogeneous boundary conditions (terms in $\eta$ and/or $\kappa$ ), and (iii) the fact that the fluxon undergoes an abrupt velocity variation at the boundaries, as specified by Eq. (22) or by Eq. (27). An operationally simple, even if by no means theoretically rigorous, way of taking fact (iii) approximately into account is to replace $\psi$ by an "average" phase-shift $\psi$ ', given by

$$
\psi^{\prime}=-\left(1-u_{b}^{2}\right)^{1 / 2} \ln u_{b}-\left(1-u_{a}^{2}\right)^{1 / 2} \ln u_{a},
$$


where $u_{b, a}$ are, respectively, the fluxon velocity before and after the reflection. For the first iteration of the map we use $L$ for the junction length. At this point, $u_{b}$ and $u_{a}$ are available from $z_{f}^{(1)}$ and $z_{0}^{(1)}$, respectively. These values are used to calculate $L_{\text {eff }}$ for the second iteration, etc. In practice, the correction of Eq. (60) gives an improved agreement with the full numerical simulation of the PDE model ${ }^{20}$ except when $u_{b, a} \ll 1$ : in such cases $\psi^{\prime}$ can become comparable with $L$, which means, from Eq. (59), that the correction no longer has sense.

A slightly more complicated, but perhaps more correct (although still certainly not rigorous) procedure is to Lorentz transform the system into a frame in which $u_{b}^{\prime}=u_{a}^{\prime}=u$, calculate the phase shift in the transformed frame by inserting this value into Eq. (58), and then Lorentz transform the phase shift back into the laboratory frame. We have not explored this approach in detail, but preliminary calculations indicate that it is somewhat less susceptible to divergence than is the correction of Eq. (60).

\section{Energy dissipation during reflections}

During a fluxon reflection in the presence of an $\alpha$-loss term, there is a momentary increase in the level of energy dissipation because of the fact that the voltage of the fluxon-antifluxon doublet is larger than that of an isolated fluxon. Pedersen et al. ${ }^{25}$ calculated this loss to be given approximately by

$$
\Delta H_{d}=-2 \pi^{2} \alpha f(u),
$$

in which $f(u)$ is a function of the velocity, which decreases smoothly from $f(0)=2$ to $f(1)=1$. Near $u=1$, Eq. (61) can be replaced by the analytic approximation ${ }^{25}$

$$
\Delta H_{d} \approx-2 \pi^{2} \alpha\left[1+4(1-u) / \pi^{2}\right] .
$$

This energy loss should thus be combined with the variation given by Eq. (14) for the overlap geometry or Eq. (20) for the in-line geometry. Olsen et al. ${ }^{17}$ demonstrated that taking into account the correction of Eq. (61), together with that due to the effect of phase shift, described earlier, yields a distinctly improved agreement, as regards the current-voltage profile of the unperturbed zero-field step, with a full numerical simulation of the PDE model.

Operationally, the simplest way of taking into account the energy dissipation during reflection is to substitute the value $u_{b}$ for $u$ in Eq. (62). More refined procedures, viz., the use of either an average or a Lorentztransformed velocity, would require the use of an iterative solution of the boundary reflection problem.

\section{DISCUSSION AND CONCLUSIONS}

The phase-locking map, described earlier, offers a relatively simple tool for studying a complex phenomenon of considerable experimental interest. Its great merit, aside from conceptual simplicity, is computational economy: with respect to full numerical simulation of the PDE system of Eqs. (2) or Eqs. (3), results from the map can be obtained with a saving of several orders of magnitude in computer time. The most significant drawback of the map derives from the hypothesis, mentioned before, that the basic dynamic configuration of the fluxon oscillator is that of a single fluxon shuttling back and forth along the junction. Physically, this is not always true, and the map obviously will predict wrong results when it is not true. In spite of this shortcoming, however, the convenience of the map is such that it seems definitely worthwhile to extend its use.

In its present form, the map offers a number of specific predictions that can be compared with experimental and numerical simulation results. In particular, we have specific predictions regarding the field-amplitude dependence of the locking range in bias current and in frequency, the qualitatively different behaviors at odd and even subharmonics and at superharmonics, the parameter dependence of hysteresis, and the existence of zerocrossing steps. An important objective for the immediate future is to perform a detailed comparison of results from the map with those from a full simulation of the PDE system, incorporating into the map the refinements discussed in Sec. VII earlier (and perhaps others) in such a way to minimize the discrepancies. At this point, an application of the map to the problem of mutual locking of oscillators in multijunction arrays would seem to be a particularly fruitful avenue to explore.

\section{ACKNOWLEDGMENTS}

We are pleased to acknowledge fruitful discussions regarding the experimental and computational aspects of phase locking with M. Cirillo, G. Costabile, R. Monaco, N. F. Pedersen, and G. Rotoli. We are grateful for the financial support received from the European Economic Comunity through Contract No. St-2-0267-J-C(A) and through the ERASMUS program, from the European Research Office of the U.S. Army through Contract No. DAJA-45-85-C-0042, from the Ministero della Pubblica Istruzione (Italy), and from the Opera Universitaria di Salerno (Italy). This work was initiated while four of us (M.S., G.F., S.P., and R.D.P) were guests of the MIDIT Center of the Technical University of Denmark, whom we thank for their hospitality.
${ }^{1}$ R. Monaco, S. Pagano, and G. Costabile, Phys. Lett. A 131, 122 (1988).

${ }^{2}$ A. K. Jain, K. K. Likharev, J. E. Lukens, and J. E. Sauvageau, Phys. Rep. 109, 309 (1984).

${ }^{3}$ R. D. Parmentier, in Solitons in Action, edited by K. Lonngren and A. Scott (Academic, New York, 1978), pp. 173-199.
${ }^{4}$ T. F. Finnegan and S. Wahlsten, in Low Temperature PhysicsLT 13, edited by K. D. Timmerhaus, W. J. O'Sullivan, and E. F. Hammel (Plenum, New York, 1974), Vol. 3, pp. 272-275.

${ }^{5}$ E. Joergensen, V. P. Koshelets, R. Monaco, J. Mygind, M. R. Samuelsen, and M. Salerno, Phys. Rev. Lett. 49, 1093 (1982). ${ }^{6}$ H. S. Newman and K. L. Davis, J. Appl. Phys. 53, 7026 (1982). 
${ }^{7}$ M. Cirillo, J. Appl. Phys. 58, 3217 (1985).

${ }^{8}$ M. Cirillo, A. R. Bishop, P. S. Lomdahl, and S. Pace, J. Appl. Phys. 66, 1772 (1989).

${ }^{9}$ M. Scheuermann, J. T. Chen, and J.-J. Chang, J. Appl. Phys. 54, 3286 (1983).

${ }^{10}$ M. Cirillo and F. L. Lloyd, J. Appl. Phys. 61, 2581 (1987).

${ }^{11}$ D. W. McLaughlin and A. C. Scott, Phys. Rev. A 18, 1652 (1978).

12J.-J. Chang, Phys. Rev. B 34, 6137 (1986).

${ }^{13}$ M. Salerno, M. R. Samuelsen, G. Filatrella, S. Pagano, and R. D. Parmentier, Phys. Lett. A 137, 75 (1989).

${ }^{14}$ J. C. Eilbeck, P. S. Lomdahl, O. H. Olsen, and M. R. Samuelsen, J. Appl. Phys. 57, 861 (1985).

${ }^{15}$ A. Barone and G. Paternò, Physics and Applications of the Josephson Effect (Wiley, New York, 1982), Chap. 5.

${ }^{16}$ G. Rotoli, G. Costabile, and R. D. Parmentier, Phys. Rev. B
41, 1958 (1990).

${ }^{17}$ O. H. Olsen, N. F. Pedersen, M. R. Samuelsen, H. Svensmark, and D. Welner, Phys. Rev. B 33, 168 (1986).

${ }^{18}$ P. L. Christiansen and O. H. Olsen, Wave Motion 2, 185 (1980).

${ }^{19}$ O. A. Levring, N. F. Pedersen, and M. R. Samuelsen, J. Appl. Phys. 54, 987 (1983).

${ }^{20}$ N. F. Pedersen and A. Davidson, Phys. Rev. B 41, 178 (1990).

${ }^{21}$ E. Ott, Rev. Mod. Phys. 53, 655 (1981).

${ }^{22}$ G. Costabile, R. Monaco, S. Pagano, and G. Rotoli (unpublished).

${ }^{23}$ R. L. Kautz and G. Costabile, IEEE Trans. Magn. MAG-17, 780 (1981).

${ }^{24}$ A. Ferrigno and S. Pace, Phys. Lett. 112A, 77 (1985).

${ }^{25}$ N. F. Pedersen, M. R. Samuelsen, and Welner, Phys. Rev. B 30, 4057 (1984). 